\title{
Genome sequence of the potato pathogenic fungus Alternaria solani HWC-168 reveals clues for its conidiation and virulence
}

\author{
Dai Zhang ${ }^{1}$, Jia-Yu He ${ }^{1}$, Parham Haddadi ${ }^{2}$, Jie-Hua Zhu ${ }^{1^{*}}$, Zhi-Hui Yang $^{1 *}$ and Lisong Ma ${ }^{1^{*}}$
}

\begin{abstract}
Background: Alternaria solani is a known air-born deuteromycete fungus with a polycyclic life cycle and is the causal agent of early blight that causes significant yield losses of potato worldwide. However, the molecular mechanisms underlying the conidiation and pathogenicity remain largely unknown.

Results: We produced a high-quality genome assembly of A. solani HWC-168 that was isolated from a major potato-producing region of Northern China, which facilitated a comprehensive gene annotation, the accurate prediction of genes encoding secreted proteins and identification of conidiation-related genes. The assembled genome of A. solani HWC-168 has a genome size $32.8 \mathrm{Mb}$ and encodes 10,358 predicted genes that are highly similar with related Alternaria species including Alternaria arborescens and Alternaria brassicicola. We identified conidiation-related genes in the genome of A. solani HWC-168 by searching for sporulation-related homologues identified from Aspergillus nidulans. A total of 975 secreted protein-encoding genes, which might act as virulence factors, were identified in the genome of A. solani HWC-168. The predicted secretome of A. solani HWC-168 possesses 261 carbohydrate-active enzymes (CAZy), 119 proteins containing RxLx[EDQ] motif and 27 secreted proteins unique to $A$. solani.
\end{abstract}

Conclusions: Our findings will facilitate the identification of conidiation- and virulence-related genes in the genome of A. solani. This will permit new insights into understanding the molecular mechanisms underlying the $A$. solani-potato pathosystem and will add value to the global fungal genome database.

Keywords: Alternaria solani, Genome sequence, Secretome, Conidiation, Virulence

\section{Background}

Alternaria, a genus of ascomycete fungi, causes various disease symptoms, including root and stem rot, blight, and wilt on most economically important plants [1]. Alternaria solani is known as the causal agent of early blight of potato and tomato. Early blight of potato is a major foliar disease that is considered one of the most destructive diseases of potato worldwide, resulting in severe yield losses in many potato growing regions [1].

\footnotetext{
*Correspondence: zhujiehua356@126.com; bdyzh@hebau.edu.cn; lisong.ma@anu.edu.au

${ }^{1}$ Center of Plant Disease and Plant Pests of Hebei Province, College of Plant Protection, Hebei Agricultural University, Baoding 071001, China Full list of author information is available at the end of the article
}

Understanding the factors influencing spore formation and identification of a wide range of secondary metabolites produced by $A$. solani have been the subject of extensive studies in the past many years. For example, Brian et al. first reported that Alternaric acid is a biologically active product of the fungus Alternaria solani $[2,3]$. In addition, $A$. solani is capable of producing extracellular polysaccharides, carbohydrases, proteases, the new zinniol-related phytotoxins $[4,5]$, and other secondary metabolites during infection [6, 7]. It has been documented that sporulation of $A$. solani depends upon many factors such as mycelial wounding, temperature, visible light, water treatment, ozone and ultraviolet [8-11]. Growth characteristics, genetic and pathogenic variations of $A$. solani have

(c) The Author(s). 2018 Open Access This article is distributed under the terms of the Creative Commons Attribution 4.0 International License (http://creativecommons.org/licenses/by/4.0/), which permits unrestricted use, distribution, and 
been studied as well [12-17]. Based on these successful and progressive studies on $A$. solani, the interaction between $A$. solani and its host represents an excellent system that will enable researchers to study the pathogenic mechanisms between Alternaria species and their hosts.

Conidiation (asexual sporulation) in filamentous ascomycetous fungi is a complex process involving the formation of conidia on conidiophores [18]. Many studies have been conducted to investigate the sporulation process, resulting in the identification of various environmental factors influencing sporulation, such as light, salt and nutrients and endogenous biological rhythms, but the light is regarded as one of the key environmental factors for regulating sporulation [19]. The molecular basis underlying the conidiation of Aspergillus nidulans and Neurospora crassa has been well studied, leading to the identification of a set of genetic regulators controlling the asexual sporulation in A. nidulans [20-22]. Activation of the transcription factor brlA gene by light has been demonstrated as an essential step of conidiation in $A$. nidulans [22, 23]. abaA gene is activated by BrlA and loss of $a b a A$ results in the formation of supernumerary tiers of cells with abacus-like structures $[18,24]$. wetA gene induced by AbaA during the late stage of conidiation activates a set of genes responsible for the synthesis of cell wall layers and spore specific functions $[25,26]$. These three sequentially expressed genes including $b r l A, a b a A$ and wet $A$ comprise a central regulatory pathway that controls the sporulation in $A$. nidulans $[18,24]$. In addition to these three genes, six upstream developmental activators ( $f l u G, f l b A, f l b B, f l b C, f l b D$ and $f(b E)$ ) have been identified by genetic studies on recessive mutations to cause defective conidiation [27]. StuA regulates transcription of the $\operatorname{brlA}$ gene and plays a key role in the structure and cell morphogenesis during the sexual and asexual phases of reproduction in $A$. nidulans $[28,29]$. Previous studies have reported that nutrition, light spectrum and temperature are major factors that influence the sporulation of $A$. solani in vitro; however, the production of conidiospores is limited and variable among distinct isolates of $A$. solani and there is no a common practical protocol developed for the species. Therefore, understanding the molecular mechanism underlying the conidiation of $A$. solani is urgently required.

Advances in next-generation sequencing (NGS) technologies are transforming biology research. The large-scale studies of fungal genome sequence have facilitated the discovery of molecular mechanisms underlying the virulence in plant fungal pathogens. Recently, several genome sequences of $A$. solani isolates have been reported including BMP0185, CBS109157 and altNL03003 [30-32]. Interestingly, the genome of $A$. solani altNL03003 isolated from a Dutch potato field has been sequenced using the long-read Pacific Biosciences (PacBio) sequencing technology. This has provided a gapless genome assembly and produced a genome size of $32.8 \mathrm{Mb}$ [32]. The available Alternaria genome sequence database provides a useful resource to browse and visualize whole genome alignments, genome annotations, and identify homologous genes within the important saprophytic and plant/human pathogenic fungal genus [18, 33-36]. However, a detailed genome annotation and prediction of genes encoding secreted proteins remain unknown for $A$. solani, especially in the genome of $A$. solan $i$ isolate from China. Here, we present an accurate genome annotation and provide a prediction of conidiation and effector candidate genes from A. solani HWC-168, which holds the potential to advance our understanding of pathogenic mechanisms of $A$. solani.

\section{Results}

\section{Genome sequencing and assembly}

To gain a better understanding of $A$. solani genome, we generated a high-quality genome sequence of $A$. solani HWC-168 using an Illumina HiSeq 2000 sequencing platform. The high quality of genomic DNA isolated from the mycelium of A. solani HWC-168 was used to prepare libraries. Two independent DNA libraries were constructed: one with insert size $500 \mathrm{bp}$ and second one with $5 \mathrm{~kb}$ insert size. Total $21.9 \mathrm{~Gb}$ and $33.7 \mathrm{~Gb}$ of high quality reads were generated from 500 bp library and $5 \mathrm{~kb}$ library, respectively. The genome coverage was 200 -fold in the library containing insert size $500 \mathrm{bp}$ and 308 -fold in the library with $5 \mathrm{~kb}$ insert size. The reads generated from both libraries were assembled into 209 contigs and 61 scaffolds, among which the size of the longest scaffold was 5,423,972 bp and scaffold N50 having the size of 2,613,338 bp. The assembled genome size achieved was $32,838,780 \mathrm{bp}$, which agrees favorably with the reported genome size of $A$. solani of 32.6 to $32.9 \mathrm{Mb}$ $[31,32]$. The GC content of the genome was $51.20 \%$ of the total bases (Table 1).

\section{Repeat content in A. solani HC-168}

To characterize the assembled genome, the repetitive elements were identified using the CENSOR prediction. In total, 24,896 repeat elements including DNA transposon, endogenous retrovirus, LTR retrotransposon, non-LTR retrotransposon, pseudogene, satellite and integrated virus were identified in the genome (Table 2). Our analysis revealed that the repeat content accounted for $6.95 \%$ of the gnome in length, which differs with that of A. solani CBS109157 [31]. The distribution of LTR retrotransposon was heavily dominant $(3.3 \%$ of the entire genome) but DNA transposon (2.43\%), Non-LTR retrotransposon $(0.91 \%)$ and endogenous retrovirus $(0.21 \%)$ were also highly represented. Based on the superfamily types, most common types of repetitive elements were represented in the $A$. solani genome with the dominant family being Gypsy (2.7\% of the genome) and the most 
Table 1 Summary of genome assembly and annotation features of A. solani, A. arborescens and A.brassicicola

\begin{tabular}{|c|c|c|c|c|}
\hline & $\begin{array}{l}\text { A. arborescens } \\
\text { EGS39-128 }\end{array}$ & $\begin{array}{l}\text { A. brassicicola } \\
\text { ATCC } 96836\end{array}$ & $\begin{array}{l}\text { A. solani } \\
\text { HWC-168 }\end{array}$ & $\begin{array}{l}\text { A. Solani } \\
\text { altNL03003 }\end{array}$ \\
\hline \multicolumn{5}{|l|}{ Genome } \\
\hline Isolate & EGS 39-128 & ATCC 96836 & HWC-168 & altNL03003 \\
\hline Coverage (fold) & 90 & 6.4 & 508 & $>150$ \\
\hline Genome size & $33,889,384 \mathrm{bp}$ & $29,536,471 \mathrm{bp}$ & $32,838,780 \mathrm{bp}$ & $32,779,142 \mathrm{bp}$ \\
\hline No. of contigs & 1332 & 4039 & 209 & 10 \\
\hline Largest contig length & $1,056,452 \mathrm{bp}$ & 87,976 bp & 1,075,562 bp & $7,235,174$ bp \\
\hline N50 contig length & 310,869 & 18,835 & 564,368 & \\
\hline G + C content $(\%)$ & 50.9 & 50.7 & 51.2 & 51.2 \\
\hline \multicolumn{5}{|l|}{ Genes } \\
\hline Number of genes & 11,042 & 10,514 & 10,358 & \\
\hline Gene density(genes/Mb) & 325 & 356 & 323 & \\
\hline Number of specific genes & 1689 & 1883 & 1632 & \\
\hline
\end{tabular}

abundant family being Copia (0.67\%) and EnSpm/CACT A $(0.60 \%)$ and Mariner/Tc1 (0.45\%).

\section{Comparison of genome assembly features within Alternaria species}

The genome size of $A$. solani HWC-168 (32.8 Mb) was small compared to $A$. arborescens $(33.9 \mathrm{Mb})$ but larger than $A$. brassicicola (29.5 Mb) [30]. It has approximately the same size as reported for $A$. solani altNL03003 $(32.8 \mathrm{Mb})$ [32]. The average gene density in A. solani HWC-168 genome was 323 genes per $\mathrm{Mb}$, which remains lower than that in A. brassicicola (356 genes per $\mathrm{Mb}$ ) and $A$. arborescens (325 genes per Mb). Next, we compared the whole genome assembly features of $A$. solani HWC-168 with those of sequenced $A$. arborescens EGS 39-128, A. brassicicola ATCC 96836 and A. solani altNL03003 genomes (Table 1). Compared to A. arborescens and $A$. brassicicola, our genome assembly was superior because it featured the greatest genome coverage, the minimum number of contigs and largest N50 contig length (Table 1). However, compared to A. solani altNL03003, a large number of contigs was observed in our assembly (Table 1). In addition, we compared the gene distribution in the three annotated $A$. brassicicola, A. arborescens and $A$. solani $H W C-168$ genomes by calculating the intergenic distance between adjacent genes. Figure 1 showed that the distributions of intergenic distances in A. solani HWC-168 genome were similar to those in the genome of $A$. brassicicola. However, the distributions of intergenic distances in $A$ arborescens genome were less variable and genes in $A$. arborescens genome were more closely spaced than the analyzed A. solani and A. brassicicola genomes (Fig. 1).

\section{Gene prediction and functional annotation}

To predict complete genes in A. solani HWC-168 genome, we used the Augustus version 2.5.5 [53]. The analysis resulted in 10,358 complete genes in the genome of A. solani HWC-168. PanOCT analysis was employed to examine the orthologous gene clusters among predicted genes of $A$. solani HWC-168, A. arborescens EGS 39-128 and A. brassicicola ATCC 96836. The total number of predicted genes, core genes, clusters of orthologous groups (COGs) and shared COGs were summarized in the Venn diagram for ortholog clusters in these three genomes (Additional file 1). The three genomes shared a core set of 3460 COGs and 6879 core genes. In addition, there were 8304 genes shared between A. solani HWC-168 and A. arborescens EGS 39-128, which was higher than that between $A$. solani HWC-168 and $A$. brassicicola ATCC 96836 (7301), and also higher than that between A. arborescens EGS 39-128 and A. brassicicola ATCC 96836 (7204). Taken together, these observations strongly indicate that significant gene variations including gene numbers and COGs exist in these closely related Alternaria strains, suggesting that these three Alternaria strains might have diverged in the genome evolution.

To annotate the predicted genes and assign Gene Ontology (GO) functions to them, predicted proteins from A. arborescens EGS39-128, A. brassicicola ATCC 96836 and A. solani HWC-168 were searched for homology to entries in the NCBI Ref Seq protein database, GO and InterPro databases using Blast2Go-PRO, respectively. As shown in Fig. 2, annotated genes contributing to the general function, amino acid transport and metabolism and carbohydrate transport and metabolism were predominant within the comparison of GO terms of three Alternaria genomes. However, further comparison of GO terms between these three Alternaria isolates revealed that the number of genes from each GO category was similar between A. solani HWC-168, A. arborescens EGS 39-128 and $A$. brassicicola ATCC 96836. In addition, we analyzed the GO functions of core genes and species-specific genes 
Table 2 Summary of repetitive elements present in the genome of A. solani HWC-168

\begin{tabular}{|c|c|c|c|c|}
\hline Repeat element & Superfamilies & Number of elements & Length occupied & $\%$ of assembled genome \\
\hline \multirow[t]{13}{*}{ DNA transposon } & & 7633 & 798,198 & 2.43 \\
\hline & EnSpm/CACTA & 1640 & 198,077 & 0.60 \\
\hline & Hobo-Activator (hAT) & 1139 & 100,772 & 0.31 \\
\hline & Mariner/Tc1 & 901 & 148,218 & 0.45 \\
\hline & MuDR & 754 & 60,332 & 0.18 \\
\hline & Harbinger & 687 & 52,748 & 0.16 \\
\hline & Polinton & 476 & 59,121 & 0.18 \\
\hline & Helitron & 374 & 27,301 & 0.08 \\
\hline & Kolobok & 233 & 33,732 & 0.10 \\
\hline & Sola & 210 & 20,091 & 0.06 \\
\hline & Crypton & 146 & 10,037 & 0.03 \\
\hline & piggyBac & 132 & 8575 & 0.03 \\
\hline & Dada & 116 & 9219 & 0.03 \\
\hline \multirow[t]{5}{*}{ Endogenous Retrovirus } & & 1069 & 69,620 & 0.21 \\
\hline & ERV1 & 414 & 27,788 & 0.08 \\
\hline & ERV2 & 360 & 23,610 & 0.07 \\
\hline & ERV3 & 102 & 6650 & 0.02 \\
\hline & ERV4 & 73 & 4385 & 0.01 \\
\hline \multirow[t]{5}{*}{ LTR Retrotransposon } & & 11,814 & $1,074,479$ & 3.30 \\
\hline & Gypsy & 6875 & 745,838 & 2.27 \\
\hline & Copia & 3378 & 220,000 & 0.67 \\
\hline & BEL & 989 & 58,924 & 0.18 \\
\hline & DIRS & 339 & 21,781 & 0.07 \\
\hline \multirow[t]{11}{*}{ Non-LTR Retrotransposon } & & 4109 & 297,677 & 0.91 \\
\hline & L1 & 1049 & 66,695 & 0.20 \\
\hline & CR1 & 538 & 44,451 & 0.14 \\
\hline & R1 & 318 & 24,513 & 0.07 \\
\hline & Jockey & 287 & 21,278 & 0.06 \\
\hline & RTE & 232 & 15,218 & 0.05 \\
\hline & $\mathrm{T} \times 1$ & 211 & 14,176 & 0.04 \\
\hline & । & 175 & 11,361 & 0.03 \\
\hline & Tad1 & 161 & 11,574 & 0.04 \\
\hline & L2 & 121 & 8125 & 0.02 \\
\hline & RTEX & 106 & 8159 & 0.02 \\
\hline \multirow[t]{3}{*}{ Pseudogene } & & 94 & 16,566 & 0.05 \\
\hline & rRNA & 57 & 13,659 & 0.04 \\
\hline & tRNA & 37 & 2907 & 0.01 \\
\hline \multirow[t]{3}{*}{ Satellite } & & 145 & 14,774 & 0.04 \\
\hline & MSAT & 40 & 6529 & 0.02 \\
\hline & SAT & 63 & 4970 & 0.01 \\
\hline \multirow[t]{3}{*}{ Integrated Virus } & & 32 & 1760 & 0.005 \\
\hline & Caulimoviridae & 29 & 1516 & 0.004 \\
\hline & DNA Virus & 3 & 244 & 0.001 \\
\hline TOTAL & & 24,896 & $2,273,074$ & 6.95 \\
\hline
\end{tabular}



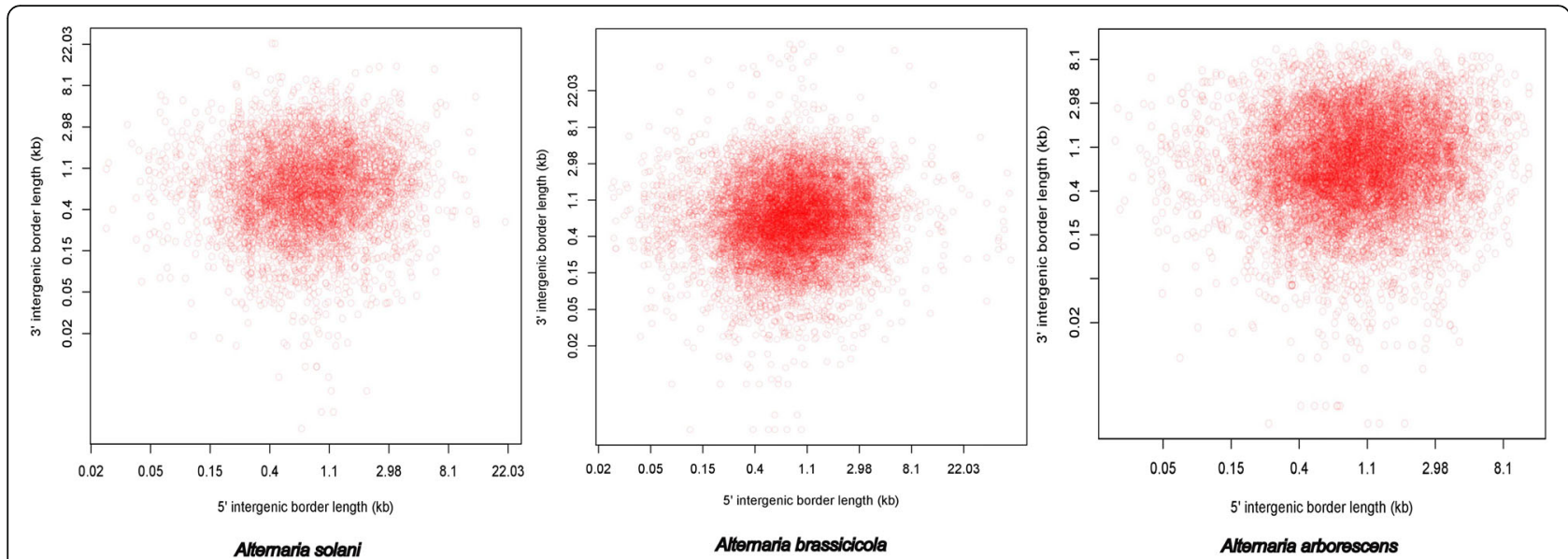

Fig. 1 Distribution of intergenic distances of all predicted genes present in the genome of A. solani HWC-168 compared with A. arborescens EGS 39-128 and A. brassicicola ATCC 96836. Scatterplot representing 5' and 3' intergenic distances for all genes present in the genome. Red circles indicate the predicted genes

between A. solani HWC-168, A. arborescens EGS 39-128 and A. brassicicola ATCC 96836. A functional GO analysis determined that core genes and species-specific genes involved in general function dominated and that the second most abundant genes were related to translation, ribosomal structure and biogenesis (Fig. 3).

\section{Secretome of $A$. solani HWC-168}

By using SignalPv4.0, TMHMM-2.0, TargetPv1.01, and big-PI Predictor, we searched the genome of $A$. solani HWC-168 for secreted protein-encoding genes, which might act as effector candidate genes. Nine hundred seventy five secreted protein-encoding genes were

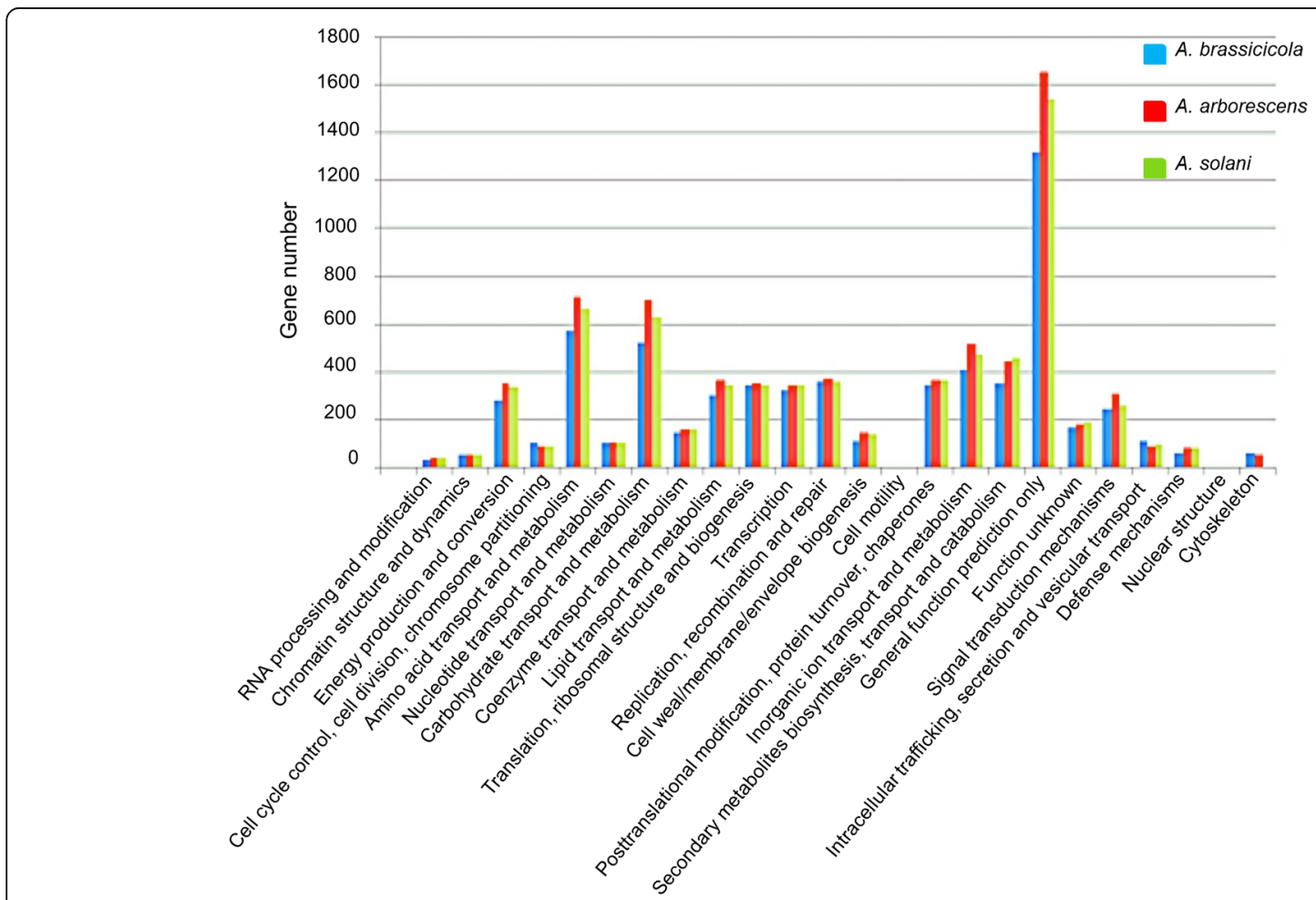

Fig. 2 Gene Ontology (GO) classification of genes predicted from the genome of A. solani HWC-168, A. arborescens EGS 39-128 and A. brassicicola ATCC 96836. Predicted genes are assigned to 24 categories in the GO classification. The $x$-axis legend shows a description of the 24 functional categories and the $y$-axis indicates the number of genes in a specific function cluster. Among the 24 categories, the cluster of 'general function prediction' has the highest number of genes, followed by amino acid transport and metabolism and carbohydrate transport and metabolism 


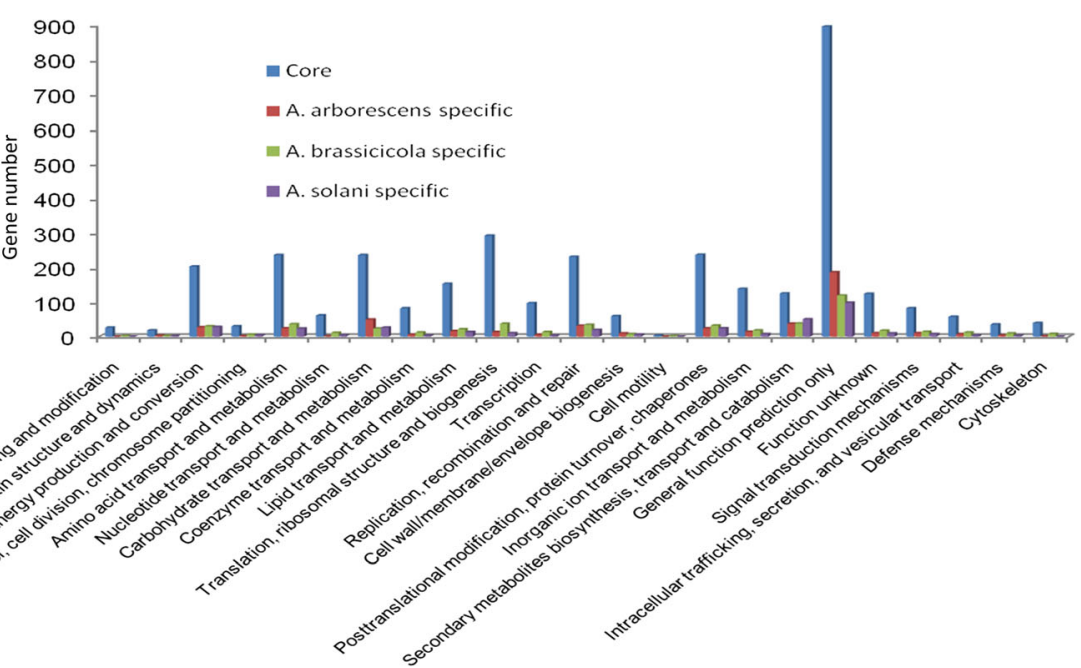

Fig. 3 Gene Ontology (GO) classification of core and species-specific genes identified from comparison of A. solani HWC-168, A. arborescens EGS 39-128 and A. brassicicola ATCC 96836 genomes. Predicted core and species-specific genes are assigned to 24 categories in the GO classification. The $x$-axis legend shows a description of the 24 functional categories and the $y$-axis indicates the number of genes in a specific function cluster. Among the 24 categories, the cluster of 'general function prediction' contains the highest number of core and species-specific genes, followed by translation, ribosomal structure and biogenesis

identified, which accounted for $9.4 \%$ of the total predicted genes.

\section{Cell wall degrading enzymes}

The majority of the secreted proteins were identified as cell wall degrading enzymes (CWDEs) involved in plant cell degradation. In addition, other enzymes that participate in various cellular metabolisms and non-enzyme proteins that maintain cellular energy and transport were also identified among these secreted proteins. Interestingly, we found that some secreted proteins identified from the secretome of $A$. solani HWC-168 were assigned to the same functional annotation but had differing functional classification (Additional file 2), suggesting that these secreted proteins may play important roles in various cellular activities.

\section{Carbohydrate-active enzymes and proteins with other predicted functions}

The A. solani HWC-168 secretome possessed 261 secreted carbohydrate-active enzymes (CAZymes) with predicted activities (Fig. 4). One protease and one SnodProt elicitor belonging to the cerato-platanin protein (CPP) family were identified within the secretome of $A$. solani HWC-168. Surprisingly, a secreted protein exhibiting sequence homology to a superoxide dismutase was identified in the secretome of $A$. solani HWC-168. It has been reported that the superoxide dismutase is involved in inhibiting oxidative damage of pathogens and plant resistance $[37,38]$. Furthermore, three trihydrophobins that are commonly found in the surface of aerial hyphae or fruiting body in fungi were predicted to be secreted $[14,15]$. The presence of trihydrophobins in the secretome of $A$. solani HWC-168 suggests their potential roles in fungal development, morphological differentiation and pathogenicity.

\section{RxLx[EDQ] effector candidates}

The RxLx[EDQ] motif functions as a host-targeting signal to deliver virulence proteins of Plasmodia falciparum into host cells [39]. The secretome of $A$. solani HWC-168 contained 119 secreted proteins possessing the $\operatorname{RxLx}[\mathrm{EDQ}]$ motif (where $\mathrm{x}$ represents any amino acid). One of important criteria for effector prediction appears to be protein size less than 300 amino acids. Based on this criterion, 12 effector candidate proteins carrying RxLx[EDQ] motif within 120 amino acids downstream of N-terminal signal peptide were identified (Table 3). WEBLOGO analysis revealed that amino acids Arginine (R) in position 1 and Leucine (L) in positon 3 and glutamic acid (E)/ aspartic acid (D)/glutamine $(\mathrm{Q})$ in the 5 position were highly conserved in the RxLx [EDQ] motif. By contrast, bilateral amino acid sequences surrounding the RxLx[EDQ] motif were not conserved and tended to be highly variable (Fig. 5a). In addition, we found that the continuous aspartic acid (D), glutamic acid $(E)$ and glutamine $(\mathrm{Q})$ residues were present in the downstream of the RxLx[EDQ] motif but with variable locations (Fig. 5b). Due to the important roles of RxLR effectors in the pathogenicity of Phytophthora infestans, functional analysis of the RxLx[EDQ] motif-containing proteins in the secretome of $A$. solani HWC-168 will be the focus for future research. 


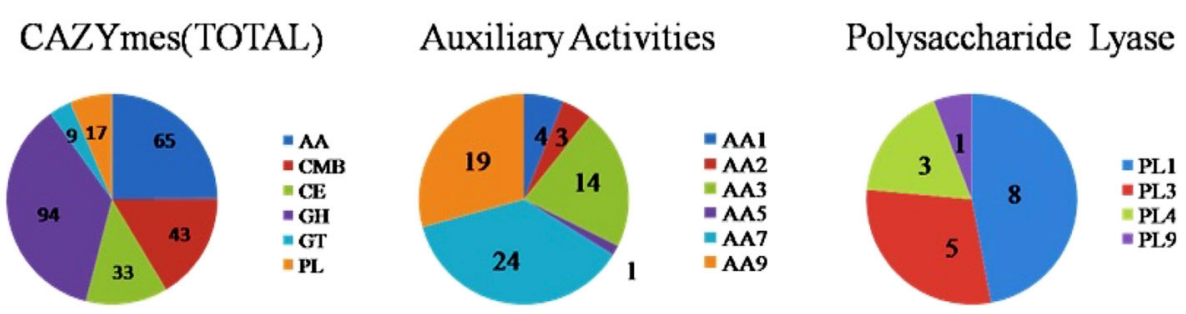

\section{Glycosyl Transferases}

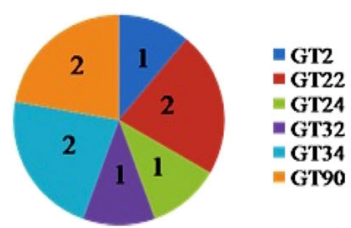

\section{Carbohydrate Esterases}

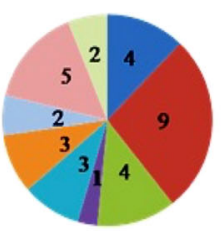

Glucoside Hydrolases

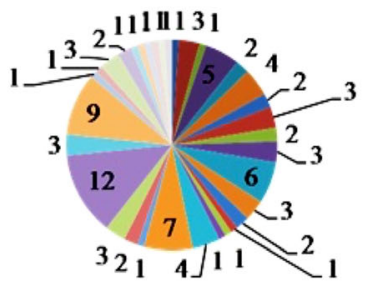

$=$ GHI $=$ GH10 GH105 $=$ GH11

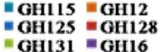
OH17 $=$ OH18 $\mathrm{GH20}=\mathrm{GH} 23$ OH26 $=\mathrm{GH} 27$ $\mathrm{OH} 28=\mathrm{OH}_{3}$ GH31 $=$ GH32 GH35 $=$ GH43 $\begin{array}{ll}\text { GH51 } & \text { GH53 } \\ \text { GH55 } & \text { GH6 }\end{array}$

\section{Carbohydrate-binding Molecules}

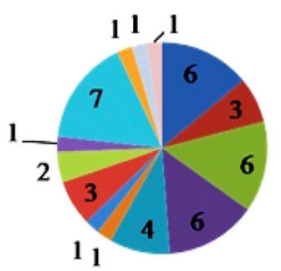

Fig. 4 Graphical representation of predicted carbohydrate-active enzymes encoding genes in the genome of A. solani HWC-168. Total 261 predicted CAZymes are identified and they are divided into six sub-groups including 65 auxiliary activity (AA), 17 polysaccharide lyase (PL), 9 glycosyl transferase (GT), 94 glucoside hydrolase (GH), 33 carbohydrate esterases (CE) and 17 carbohydrate-binding molecules (CBM). Glucoside hydrolase is predominant in all predicted CAZymes

\section{Unique secreted proteins}

We observed that 27 predicted genes encoding secreted proteins were completely absent in the genome of $A$. arborescens and $A$. brassicicola (Additional file 3 ). As a consequence, the function of these secreted proteins remains unknown due to the lack of homology with known proteins. Interestingly, from 27 species-specific genes we identified 3 pairs of neighbor genes that reside on three different scaffolds: scaffold 18, scaffold 21 and scaffold 8, respectively (Additional file 4). These findings suggest that the presence of these species-specific secreted protein-encoding genes in the genome of $A$. solani HWC-168 may have originated by two possibilities: either the genome of $A$. solani HWC-168 possesses

Table 3 List of predicted effector candidates carrying the RxLx[EDQ] motif

\begin{tabular}{|c|c|c|}
\hline Name & Size & Start of sequence \\
\hline ASLO_253 & 148 & MKFTLAIVALASVATTALANREWTYNDSHRAAVTAILKQITAKHAHLCKR \\
\hline ASLO_323 & 134 & MKLAIAALFASITAAAPTATPDVHGDPFETVTISNFVYVGVNGYPQIDFH \\
\hline ASLO_2101 & 114 & MQIMNLAVLAATLATVGAWTLDDYGKWVANNAWRDNLNGVHKVHESCAER \\
\hline ASLO_4082 & 266 & MKFLAIIVAAQLATALPVAKEACSSTDITCSATKANPQIFDVASQQLDNS \\
\hline ASLO_5583 & 205 & MVSFRNLFTAAMALSVPVAAVLTPAQIVDNIRILTQKSQALQAPAQSITI \\
\hline ASLO_5663 & 199 & MASSIPDHWLWLGLGVFTFIAVQQVSHGLHTIRALTEIRNPQNMPQQRRA \\
\hline ASLO_6610 & 169 & MLGRTVFAATFFALAQFAMASPPSCLLGAVNQYEDPSDIKAVCKARNLSE \\
\hline ASLO_6668 & 183 & MFLPTALLALVHLALPALSHASPQPALVSSDWEMSLVPRHQLFLRQLSDL \\
\hline ASLO_8389 & 252 & MYSKTAIVTFFAGFAAAQIHAPVGEPSGNPITRPLNEWPTCEQFTITWQ \\
\hline ASLO_8909 & 270 & MAKLIDLSTEVLFLIVAYFTSGDASDVQALLHLCRTSRMLVAVAQPALYT \\
\hline ASLO_9385 & 163 & MHFSVIFSAVFAATAMAAPASLDSRGDEDCVPDSYTISDYTLITSPTSGS \\
\hline ASLO_10144 & 244 & MLSNLMNRFALPLAILAFFLSFANGLPHDALIARRTTNLRILPLGDSITW \\
\hline
\end{tabular}




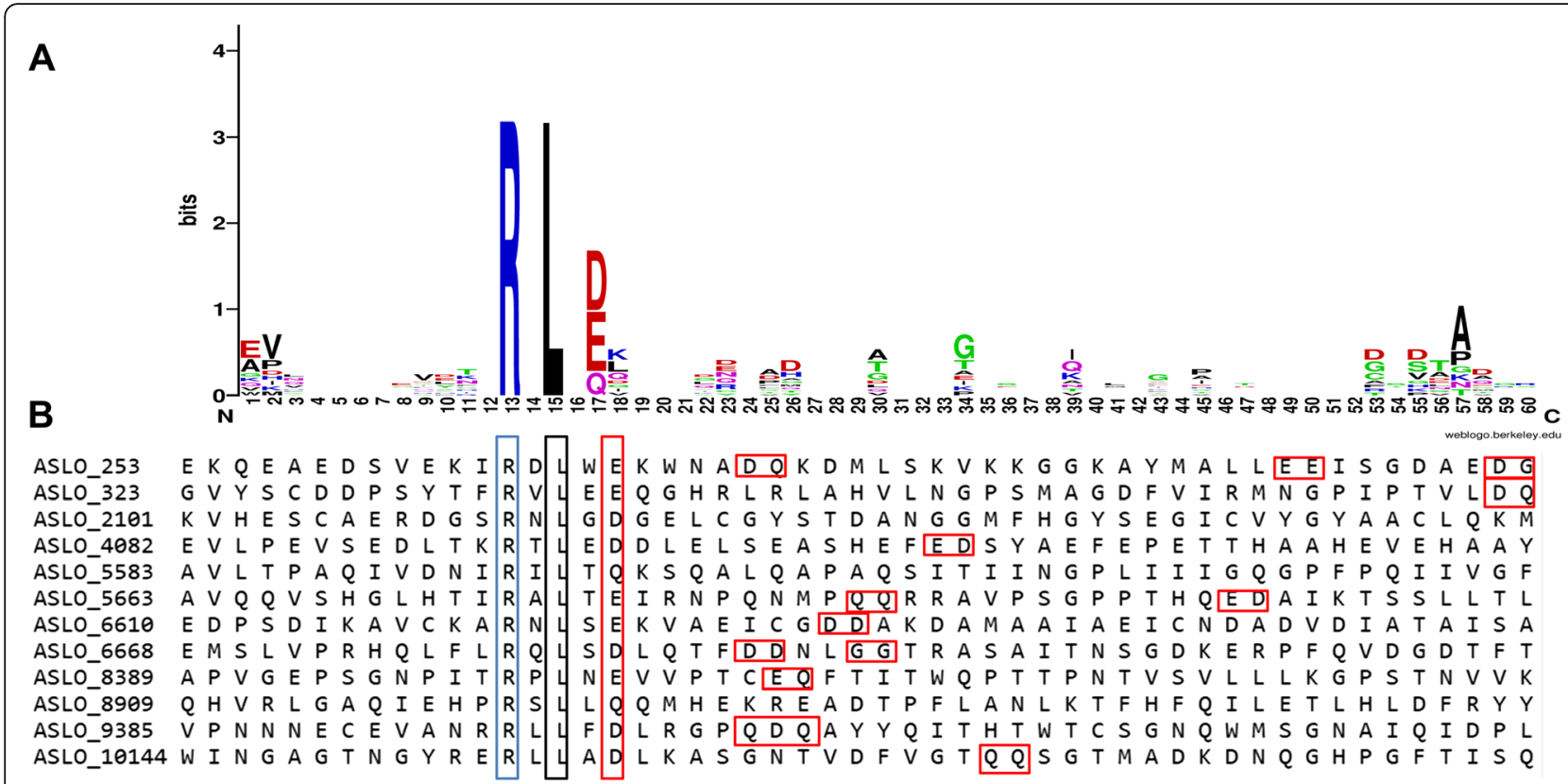

Fig. 5 Schematic representation of amino acid sequences alignment of 12 RxLx-motif containing effector candidates. a. Sequence logo derived from 12 predicted secreted effector candidates carrying the RxLx[EDQ] motif located within the region of 120 amino acids downstream of the Nterminal signal peptide. $\mathbf{b}$. the conserved amino acids in the RxLx[EDQ] motif are highlighted and the downstream EDQ amino acids are marked with a red rectangle

a large genomic fragment that is missing in other Alternaria genomes or the genomic databases of $A$. arborescens and $A$. brassicicola are incomplete because only their draft genomes are reported, which has resulted in the absence of these secreted proteins. Functional analysis of these species-specific secreted proteins in $A$. solani HWC-168 is under way in our laboratory.

\section{Prediction of conidiation-related genes}

Our earlier studies revealed that it was difficult to induce conidiation in our A. solani isolates under artificial culturing condition. However, A. solani HWC-168 is capable of yielding copious conidiospores when its mycelia are radiated by ultraviolet (UV light) (Additional file 5). To identify genes involved in conidiation, we retrieved the central regulatory genes participating in the conidiation from the genome of Aspergillus nidulans and blasted the $\operatorname{brl} A$ sequence and other sporulation related genes, such as abaA, wetA, stuA, fluG, flbA, flbC, flbD, flbE, medA and $\operatorname{fad} A$, to the predicted protein database of $A$. solani. Our results showed that homologous genes including $f l u G, f l b A, f l b C, f l b E, b r l A, s t u A, a b a A$, wetA, medA and $\mathrm{fadA}$ existed in the genome of A. solani-HWC168. Based on the proposed conidiation model in Aspergillus nidulans and the identified conidiation-related genes in $A$. solani HWC-168, we propose a potential conidiation pathway in A. solani HWC-168 (Fig. 6).

\section{Discussion}

Pathogenic A. solani strains are increasingly posing a critical threat to world food security. Sequencing the whole genome of $A$. solani is a key step to facilitate the study of the molecular mechanisms underlying the interaction between potato and A. solani. Here, we presented the completed genome sequence and annotation of $A$. solani HWC-168 generated by advanced next-generation Illumina sequencing technology. The quality of the genome sequence was guaranteed by using two individual sequencing libraries. The genome sequence data of $A$. solani $\mathrm{HWC}-168$ has the potential to facilitate a future study on the molecular basis of $A$. solani virulence.

Genomes of three $A$. solani isolates have been sequenced in the past [30-32]. The assembled genome size of $A$. solani ranges from 32.6 to $32.9 \mathrm{Mb}$. Our sequenced $A$. solani HWC-168 produced a genome size of $32.8 \mathrm{Mb}$, which compares favorably with the reported genome size of $A$. solani altNL03003 (Table 1). The genome of $A$. solani altNL03003 had been sequenced using the long-read Pacific Biosciences (PacBio) sequencing technology, which provided a gapless genome assembly. Although we sequenced the genome of $A$. solani HWC-168 using the second-generation sequencing technology, the same genome size was produced with $A$. solani altNL03003 but the assembled contig number of $A$. solani HWC-168 being higher than that of $A$. solani altNL03003. This suggests a high-quality genome assembly of altNL03003 has 


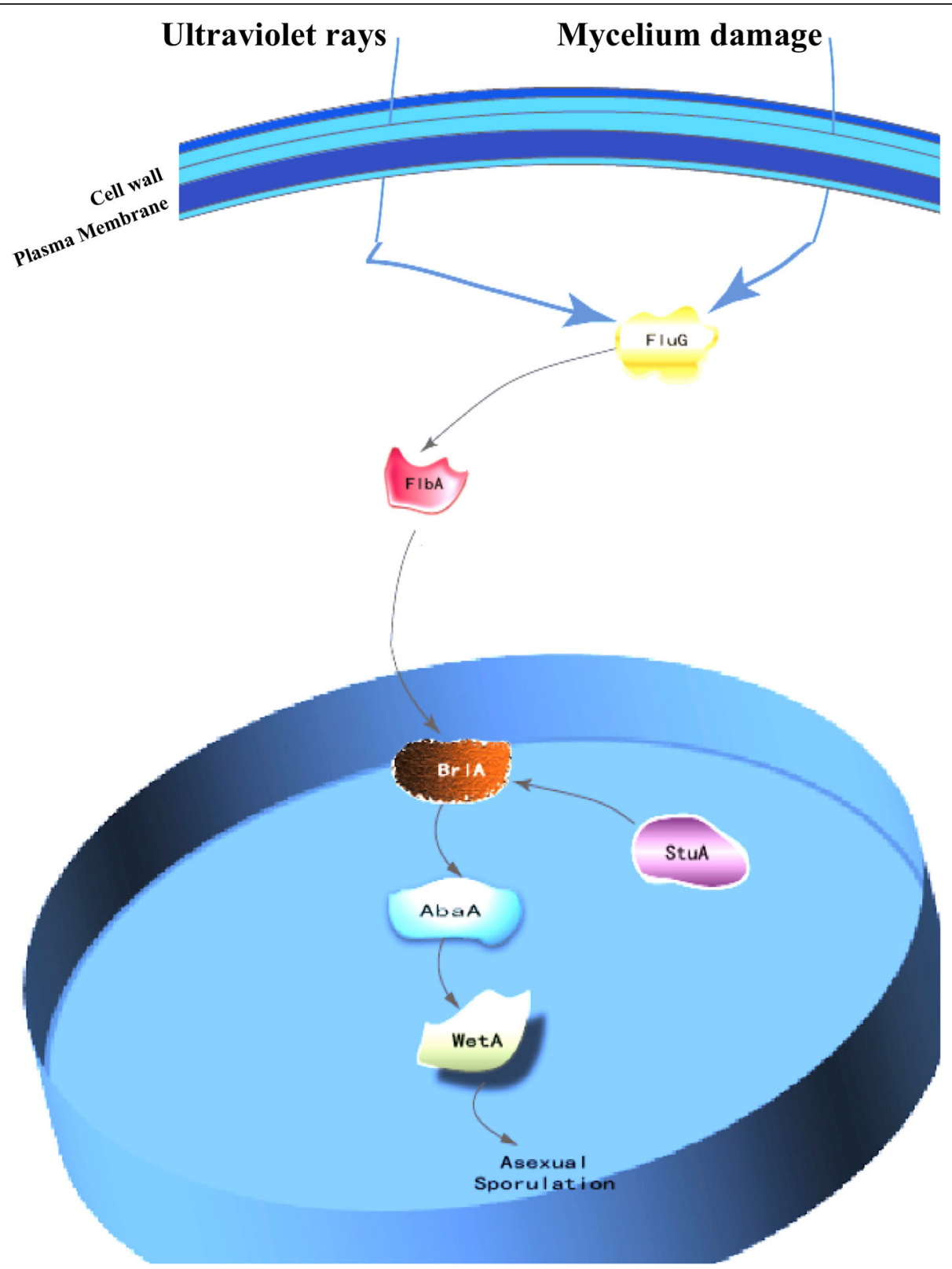

Fig. 6 Schematic representation of a proposed model illustrating the regulatory pathway of asexual sporulation in A. solani

been obtained. However, we were not able to compare our predicted genes with those of $A$. solani altNL03003 because of the lack of annotation data of $A$. solani altNL03003. By searching our predicted RxLx[EDQ] effector candidates and conidiation-related genes in the genome of $A$. solani altNL03003, we found that all of them were present in the genome of $A$. solani altN L03003 (Additional file 3 and unpublished data). This observation strongly suggests that the genome annotation of A. solani HWC-168 is accurate. It has been reported that the repeat content in the genome of $A$. solani CBS109157 is relatively low with only $1.5 \%$ [31]. Surprisingly, we found that the percentage of repeat content of $A$. solani HWC-168 was relatively high with $6.95 \%$ although both of them have similar genome size. This apparent discrepancy requires future study to identify repeat contents from the gapless assembled genome of A. solani altNL03003.

Asexual sporulation is a common reproduction strategy in filamentous fungi. Although they vary in morphology and function, conidia in higher fungi are developed from specialized sporogenous cells or asexual propagules. The 
conidiation-related processes are complicated, including the space-time regulation of conidiation-related genes, cell specialization and cell signal transduction, etc. The genetic mechanism of conidiation in Aspergillus nidulans has been well studied [40, 41]. However, the molecular basis underlying conidiation in A. solani remains unclear. Elucidation of genes regulating the conidiation process is essential to our understanding of asexual reproduction in $A$. solani species. Here, we first presented the evidence that the central regulatory factors of conidiation identified from Aspergillus. nidulans including abaA, wetA, StuA, FluG and FlbA genes are present in the genome of $A$. solani HWC-168. This suggests a similar molecular mechanism of sporulation is employed by $A$. solani; however, these homologous conidiation-related genes in A. solani are putative and functional confirmation is required. We are examining the expression profiles of predicted conidiation-related genes (unpublished data). We are confident that future studies designed to elucidate the molecular basis of conidiation will provide the impetus to develop novel strategies to prevent sporulation in order to control disease development caused by $A$. solani on potato.

The secreted fungal enzymes in pathogenic fungi play important roles in pathogenicity. Here, we found that the secreted enzymes in $A$. solani HWC-168 contain a large number of cellulases and pectinases. Previous studies showed that cellulases and pectinases in Alternaria species play key roles in degrading the plant cell wall during infection [42-44]. Thus, we proposed that cellulases and pectinases in $A$. solani play important roles in infecting the host and causing degradation of host cell wall. In our work, a total of 975 predicted secreted proteins were identified in the genome of $A$. solani HWC-168, comprising 261 CAZymes, 119 RxLx[EDQ] motif containing secreted proteins and 27 species-specific secreted proteins. It remains unknown how these species-specific secreted proteins contribute to the pathogenicity of $A$. solani HWC168. However, we speculate that some of the proteins might function inside plant cells, which has been widely reported in the effector proteins of rust and oomycete plant pathogens [45-48]. Translocation of rust and oomycete effector proteins into plant cell largely depends on the conserved RxLx-motif $[46,47]$. In the secretome of A. solani HWC-168, 12 RxLx-motif containing effector candidate proteins were found, which indicates that they might serve as virulence factors during $A$. solani infection. Recent reports showed that RxLR effectors from various fungal pathogens are involved in virulence, which will broaden the implications of our findings [49]. The RxLx-motif containing effectors involving in the pathogenicity during the interaction between A. solani HWC-168 and potato will be further investigated.

\section{Conclusions}

In this study we developed and annotated the complete genome sequence of $A$. solani HWC-168, and predicted the conidiation-related genes and the secretome that contains the virulence-related genes. To our best knowledge, this is the first time that the effector candidate genes and conidiation-related genes have been predicted in the genome of $A$. solani, which will facilitate the identification and functional analysis of conidiationand virulence-related genes in A. solani. Availability of the genome sequence of $A$. solani HWC-168 and its host potato coupled with advanced genetic and molecular approaches will enable an understanding of the molecular mechanisms underlying the $A$. solani-potato pathosystem.

\section{Methods}

\section{Strain and culture conditions}

The strain A. solani HWC-168 was isolated from the infected leaves of potato in Weichang County Hebei province in China. All Alternaria isolates were cultured on potato dextrose agar (PDA). Mycelia were obtained by growing the isolate for at least 7 days on PDA plates.

\section{Genomic DNA preparation and library construction}

The mycelia were harvested by filtration and frozen at $-20{ }^{\circ} \mathrm{C}$. DNA was extracted from the mycelia according to a modified etyltrimethylammonium bromide procedure (CTAB) [50]. Following DNA fragmentation, we constructed two genomic sequencing libraries: one is a paired-end library with $500 \mathrm{bp}$ inserts and another is a mate-pair library with $5 \mathrm{kp}$ insertion fragments. The paired-end library was constructed using the Paired-End DNA sample Prep Kit (Illumina, USA) following the protocols provided by the manufacturer. The mate-pair library was constructed using the Nexera Mate Pair Library Prep Kit (Illumina, USA) following the protocols provided by the manufacturer.

\section{Genome sequencing and assembly}

Two constructed libraries were sequenced by using the Illumina GA II technology (Illumina, USA) Hiseq 2000 platforms at the Beijing Genomics Institute using the WGS (whole genome sequencing) method. The read length is $150 \mathrm{bp}$. Low-quality data containing a quality value of less than 20 and short reads (length $35 \mathrm{bp}$ ) were filtered from raw data by Dynamic Trim and Length Sort Perl program in the SolexaQA software [51]. SOAPdenovo software (http://soap.genomics.org.cn) was used to assembly sequences and gaps were immerged by SOAP Gap Closer software (http://soap.genomics.org.cn) [52]. ORFs (open reading frames) were predicted by Augustus 2.5.5 software [53], and were aligned with homologous proteins 
in the NCBI database (http://www.ncbi.nlm.nih.gov/). All confirmed ORFs were aligned with COG (Clusters of Orthologous Groups) in the NCBI database, and were classified by function based on alignment results and classification standards of COG. Repetitive elements were identified by CENSOR (http://www.girinst.org/censor) following the default parameters.

\section{Genome comparison}

The genome comparison was performed among $A$. solani HWC-168, A. arborescens and A. brassicicola. Multiple sequence alignments of genomes were performed with Mugsy [54]. The homologous genes were aligned using PanOCT software [55] by designating the parameter values: protein sequence with $>60 \%$ identity, aligned length $>30 \%$ and $E$ value less than $1 e-5$, the Intergenic distance was calculated using the method described previously [56].

\section{Secreted protein annotation and prediction}

The secreted proteins putatively encoded in the genome of $A$. solani HWC-168 were predicted by SignalPv4.0 (http://www.cbs.dtu.dk/services/SignalP/), TMHMM-2.0 (http://www.cbs.dtu.dk/services/TMHMM-2.0/), TagetPV1.01 (http://www.cbs.dtu.dk/services/TargetP/), and Big-pi (http:// mendel.imp.ac.at/gpi/fungi_server.html). In detail, we followed the effector prediction pipeline described previously [57]. We first searched the ones with the presence of an N-terminal signal peptide through signalIP4.1. Then, we excluded the ones with a predicted transmembrane domains using TMHMM-2.0. Next, we detected the presence of subcellular localization signals using TargetP and glycosylphophatidylinositol (GPI) anchor to the membrane and filtered out the ones with mitochondrial localization and then the ones with GPI. The secreted proteins in A. arborescens and A. brassicicola were predicted by using the same method. The secreted proteins in $A$. solani HWC-168 were used as inquiring sequences to search against the secretomes of $A$. arborescens and A. brassicicola by BLASTP. The parameter values were designated as E-value $<10^{-5}$ and identity $>$ $30 \%$. The secreted proteins with no homologous ones in $A$. arborescens and $A$. brassicicola were defined as species-specific proteins in A. solani HWC-168.

\section{Carbohydrate-active enzyme annotation}

All putative proteins of A. solani HWC-168 were searched against entries in the CAZy database by using CAZymes Analysis Toolkit [58] using the Carbohydrate Active Enzymes (CAZy) database (http://www.cazy.org). The parameter values were in default on the website. All identified proteins were then manually retrieved.

\section{Prediction of proteins with a $\operatorname{RxLx}[\mathrm{EDQ}]$}

The predicted secreted proteins in A. solani HWC-168 were examined with the presence of the conserved host-targeting motif RxLx[EDQ]by using the MEME prediction server (http://meme-suite.org/tools/meme) with default parameters. The amino acids in the conserved $\operatorname{RxLx}[E D Q]$ motif were aligned by WEBLOGO software [59].

\section{Additional files}

Additional file 1: Venn-diagram showing the cluster of orthologous group (COGs) genes for related three strains including A. solani, A. arborescens and A. brassicicola. Ortholog clusters were computed by using PanOCT with set parameter cutoffs (E value $<10^{-5}$; match length $>30 \%$; identity $>60 \%$ ). (DOCX $137 \mathrm{~kb}$ )

Additional file 2: Representative enzymes with the same function but involving in different biological activities. (DOCX 14 kb)

Additional file 3: Twenty seven species-specific secreted proteins based on the prediction. (DOCX $17 \mathrm{~kb}$ )

Additional file 4: Three pairs of specific neighbor genes reside on three different scaffolds. (DOCX $14 \mathrm{~kb}$ )

Additional file 5: Conidia and conidiophores formed by A. solani HWC168 were visualized under microscopy. (DOCX $154 \mathrm{~kb}$ )

\section{Abbreviations \\ CAZy: Carbohydrate-active enzymes; COG: Clusters of orthologous groups; CPP: Cerato-platanin protein; CTAB: Etyltrimethylammonium bromide; CWDEs: Cell wall degrading enzymes; GPI: Glycosylphophatidylinositol}

\section{Acknowledgments}

We thank Dr. Gordon Gropp (Saskatoon Development and Research Centre of Agriculture and Agri-Food Canada) and Dr. Likui Zhang (Yangzhou University) for the critical reading of our manuscript.

\section{Funding}

This research was supported by the Earmarked Fund for Modern Agro-industry Technology Research System (CARS-09-P18), the National Key Research and Development Program of China (2018YFD0200806) and The Earmarked Fund for Modern Agro-industry Technology Research System in Hebei Province, China (HBCT2018080205). The funding body has no role in the design of the study and collection, analysis and interpretation and in writing the manuscript.

\section{Availability of data and materials}

The datasets including genome sequence and assembly are available in NCBI GenBank under accession number JRWV00000000.1. The datasets including predicated genes and conidiation-related genes are available from the corresponding author upon reasonable request. The rest of datasets generated or analyzed in this work are included in this published article. Strains were collected and taken according to the guidelines of the Chinese "Biosafety Management Regulations for Pathogenic Microbiological Laboratory".

\section{Authors' contributions}

$D Z, J H Z, Z H Y$ and LM conceived and designed the experiments. DZ, JYH and $\mathrm{PH}$ performed the experiments and $\mathrm{PH}, J \mathrm{HZ}, \mathrm{LM}$ and $\mathrm{ZHY}$ analyzed the data. $\mathrm{DZ}, \mathrm{LM}, \mathrm{JHZ}$ and $\mathrm{ZHY}$ drafted the manuscript. All authors read and approved the final manuscript.

Ethics approval and consent to participate Not applicable

Consent for publication

Not applicable

Competing interests

The authors declare that they have no competing interests. 


\section{Publisher's Note}

Springer Nature remains neutral with regard to jurisdictional claims in published maps and institutional affiliations.

\section{Author details}

${ }^{1}$ Center of Plant Disease and Plant Pests of Hebei Province, College of Plant Protection, Hebei Agricultural University, Baoding 071001, China. ${ }^{2}$ Saskatoon Research and Development Centre, Agriculture and Agri-Food Canada, Saskatoon, SK S7NOX2, Canada.

\section{Received: 8 May 2018 Accepted: 22 October 2018} Published online: 06 November 2018

\section{References}

1. Haware MP. Assessment of losses due to early blight (Alternaria solani) of potato. Mycopathol Mycol Appl. 1971;43(3):341-2.

2. Brian PW, Curtis PJ, Hemming HG, Wright JM. Alternaric acid, a biologically active metabolic product of the fungus Alternaria solani. Nature. 1949;164(4169):534

3. Brian PW, Curtis PJ, Hemming HG, Jefferys EG, Wright JM. Alternaric acid; a biologically active metabolic product of Alternaria solani (Ell. \& Mart.) Jones \& Grout; its production, isolation and antifungal properties. J Gen Microbiol. 1951:5(4):619-32.

4. GamboaAngulo MM, AlejosGonzalez F, PenaRodriguez LM. Homozinniol, a new phytotoxic metabolite from Alternaria solani. J Agric Food Chem. 1997; 45(1):282-5

5. Moreno-Escobar J, Puc-Carrillo A, Caceres-Farfan M, Pena-Rodriquez LM Gamboa-Angulo MM. Two new zinniol-related phytotoxins from Alternaria solani. Nat Prod Res. 2005;19(6):603-7.

6. Ai HL, Zhang LM, Chen YP, Zi SH, Xiang H, Zhao DK, Shen Y. Two new compounds from an endophytic fungus Alternaria solani. J Asian Nat Prod Res. 2012:14(12):1144-8.

7. Andersen B, Dongo A, Pryor BM. Secondary metabolite profiling of Alternaria dauci, A. porri, A. solani, and A. tomatophila. Mycol Res. 2008;112:241-50.

8. Prasad B, Dutt BL. Inducing sporulation in Alternaria solani. II. Effect of light. Mycopathol Mycol Appl. 1974;54(1):47-54

9. Prasad B, Dutt BL, Nagaich BB. Inducing sporulation in Alternaria solani. I. Effect of water treatment. Mycopathol Mycol Appl. 1973;49(2):141-6.

10. Rich S, Tomlinson H. Effects of ozone on conidiophores and conidia of alternaria solani. Phytopathology. 1968:58(4):444-6.

11. Singh BM. Inducing sporulation in different strains of Alternaria solani. II. Effect of ultraviolet light. Mycopathol Mycol Appl. 1967;32(2):163-71.

12. Leiminger $\mathrm{JH}$, Auinger $\mathrm{HJ}$, Wenig $\mathrm{M}$, Bahnweg $\mathrm{G}$, Hausladen $\mathrm{H}$. Genetic variability among Alternaria solani isolates from potatoes in southern Germany based on RAPD-profiles. J Plant Dis Protect. 2013;120(4):164-72.

13. Lourenco V, Rodrigues TTM, Campos AMD, Braganca CAD, Scheuermann KK, Reis A, Brommonschenkel SH, Maffia LA, Mizubuti ESG. Genetic structure of the population of Alternaria solani in Brazil. J Phytopathol. 2011;159(4):233-40.

14. Tymon L, Cummings TF, Johnson DA. Pathogenicity and aggressiveness of Alternaria solani, A. alternata, and A. triticina on potato. Phytopathology. 2013;103(6):149-50.

15. van der Waals JE, Korsten L, Slippers B. Genetic diversity among Alternaria solani isolates from potatoes in South Africa. Plant Dis. 2004;88(9):959-64.

16. Varma PK, Singh H, Gandhi SK, Chaudhary K. Variability among Alternaria solani isolates associated with early blight of tomato. Commun Agric Appl Biol Sci. 2006;71(4):37-46.

17. Weber B, Halterman DA. Analysis of genetic and pathogenic variation of Alternaria solani from a potato production region. Eur J Plant Pathol. 2012; 134(4):847-58.

18. Park HS, Yu JH. Genetic control of asexual sporulation in filamentous fungi. Curr Opin Microbiol. 2012;15(6):669-77.

19. Bahn YS, Xue H, Idnum A, Rutherford JC, Heitman J, Cardenas ME. Sensing the environment: lessons from fungi. Nat Rev Microbiol. 2007;5:57-69.

20. Etxebeste O, Garzia A, Espeso EA, Uqalde U. Aspergillus nidulans asexual development: making the most of cellular modules. Trends Microbiol. 2010; 17(12):569-72.

21. Son H, Kim MG, Min K, Seo YS, Lim JY, Choi GJ, Kim JC, Chae SK, Lee YW. AbaA regulates conidiogenesis in the ascomycete fungus Fusarium graminearum. PLoS One. 2013:8(9):e72915.

22. Adams T, Boylan MT, Timberlake WE. BrlA is necessary and sufficient to direct conidiophore development in Aspergillus nidulans. Cell. 1988;54(3): 353-62.
23. Ruger-Herreros C, Rodríguez-Romero J, Fernández-Barranco R, Olmedo M, Fischer R, Corrochano LM, Canovas D. Regulation of conididation by light in Aspergillus nidulans. Genetics. 2011;188(4):809-22.

24. Mirabito PM, Adams TH, Timberlake WE. Interactions of three sequentially expressed genes control temporal and spatial specificity in aspergillus development. Cell. 1989:57(5):859-68.

25. Sewall TC, Mims CW, Timberlake WE. Conidium differentiation in Aspergillus nidulans wild-type and wet-white (wetA) mutant strains. Dev Biol. 1990; 138(2):499-508.

26. Marshall MA, Timberlake WE. Aspergillus nidulans wetA activates sporespecific gene expression. Mol Cell Biol. 1991;11(1):55-62.

27. Wieser J, Lee BN, Fondon JW, Adams TH. Genetic requirements for initiating asexual development in Aspergillus nidulans. Curr Genet. 1994;27(1):62-9.

28. Wu J, Miller BL. Aspergillus asexual reproduction and sexual reproduction are differentially affected by transcriptional and translational mechanisms regulating stunted gene expression. Mol Cell Biol. 1997;17(10):6191-201.

29. Miller KY, Wu J, Miller BL. StuA is required for cell pattern formation in aspergillus. Genes Dev. 1992;6(9):1770-82

30. Dang HX, Pryor B, Peever $\mathrm{T}$, Lawrenc CB. The Alternaria genomes database: a comprehensive resource for a fungal genus comprised of saprophytes, plant pathogens, and allergenic species. BMC Genomics. 2015;16:239-50.

31. Woudenberg JHC, Seidl MF, Groenewald JZ, de Vries M, Stielow JB, Thomma BPHJ, Crous PW. Alternaria section Alternaria: species, formae speciales or pathotypes? Stud Mycol. 2015;82:1-21.

32. Wolters PJ, Faino L, van den Bosch TBM, Evenhuis B, Visser RGF, Seidl MF, Vleeshouwers VGAA. Gapless genome assembly of the potato and tomato early blight pathogen Alternaria solani. Mol Plant-Microbe Interact. 2018; 31(7):692-4.

33. Huang K, Zhong Y, Li Y, Zheng D, Cheng ZM. Genome-wide identification and expression analysis of the apple ASR gene family in response to Alternaria alternata f. sp mali. Genome. 2016;59(10):866-78.

34. Bihon W, Cloete M, Gerrano AS, Oelofse D, Adebola P. Draft genome sequence of Alternaria alternata isolated from onion leaves in South Africa. Genome Announc. 2016:4(5):1022-16.

35. Nguyen HD, Lewis $C T$, Levesque CA, Grafenhan T. Draft genome sequence of Alternaria alternata ATCC 34957. Genome Announc. 2016:4(1):1554-15.

36. Wang M, Sun X, Yu D, Xu J, Chung K, Li H. Genomic and transcriptomic analyses of the tangerine pathotype of Alternaria alternata in response to oxidative stress. Sci Rep. 2016;6(1):1-11.

37. Lightfood DJ, Mcgrann GR, Able AJ. The role of cytosolic superoxide dismutase in barley-pathogen interactions. Mol Plant Pathol. 2017;18(3):323-35.

38. Lu F, Liang X, Lu H, Li Q, chen Q, Zhang P, Li K, Liu G, Yan W, Song J, Duan C, Zhang L. Overproduction of superoxide dismutase and catalase confers cassava resistance to Tetranychus cinnabarinus. Sci Rep. 2017;7:40179.

39. Marti M, Good RT, Rug M, Knuepfer E, Cowman AF. Targeting malaria virulence and remodeling proteins to the host erythrocyte. Science. 2004;10: 1930-3.

40. Emri T, Molnar Z, Pusztahelyi T, Varecza Z, Pocsi I. The fluG-BrlA pathway contributes to the initialisation of autolysis in submerged Aspergillus nidulans cultures. Mycol Re. 2005;109:757-63.

41. Rodrigues TTMS, Maffia LA, Dhingra OD, Mizubuti ESG. In vitro production of conidia of Alternaria solani. Trop Plant Pathol. 2010;35(4):203-12.

42. Goatley JL. Production of exocellular polysaccharides by Alternaria solani. Can J Microbiol. 1968;14(10):1063-8.

43. Shahbazi $H$, Aminian $H$, Sahebani N, Halterman DA. Activity of beta-1,3glucanase and beta-1,4-glucanase in two potato cultivars following challenge by the fungal pathogen Alternaria solani. Phytoparasitica. 2011; 39(5):455-60.

44. Cho Y, Jang M, Srivastava A, Jang JH, Soung NK, Ko SK, Kang DO, Ahn JS, Kim BY. A pectate lyase-coding gene abundantly expressed during early stages of infection is required for full virulence in Alternaria brassicicola. PLoS One. 2015:10(5):e0127140.

45. Kemen E, Kemen AC, Rafigi M, Hempel U, Mendgen K, Hahn K, Hahn M, Voegele RT. Identification of a protein from rust fungi transferred from haustoria into infected plant cells. Mol Plant-Microbe Interact. 2005;18(11): $1130-9$

46. Dodds PN, Lawrence G, Catanzariti AM, Ayliffe MA, Ellis JG. The Melampsora lini AvrL567 avirulence genes are expressed in haustoria and their products are recognized inside plant cells. Plant Cell. 2004;16(3):755-68.

47. Birch PRJ, Rehmany AP, Pritchard L, Kamoun S, Beynon JL. Trafficking arms: oomycete effectors enter host plant cells. Trends Microbiol. 2006;14(1):8-11. 
48. Mendgen K, Hahn M. Plant infection and the establishment of fungal biotrophy. Trends Plant Sci. 2002;7(8):352-6.

49. Choi J, Park J, Kim D, Jung K, Kang S, Lee YH. Fungal Secretome database: integrated platform for annotation of fungal secretomes. BMC Genomics. 2010;11:105.

50. Storchova H, Hrdlickova R, Chrtek J, Tetera M, Fitze D, Fehrer J. An improved method of DNA isolation from plants collected in the field and conserved in saturated $\mathrm{NaCl} / \mathrm{CTAB}$ solution. Taxon. 2000;49(1):79-84.

51. Cox MP, Peterson DA, Biggs PJ. SolexaQA: at-a-glance quality assessment of Illumina second-generation sequencing data. BMC Bioinformatics. 2010;11:485.

52. Luo R, Liu B, Xie Y, Li Z, Huang W, Yuan J, He G, Chen Y, Pan Q, Liu Y, Tang J, Wu G, Zhang H, Shi Y, Liu Y, Yu C, Wang B, Lu Y, Han C, Cheung DW, Yiu SM, Peng S, Xiaogian Z, Liu G, Liao X, Li Y, Yang H, Wang J, Lam TW, Wang J. SOAPdenovo2: an empirically improved memory-efficient short-read de novo assembler. Gigascience. 2012;1(1):18.

53. Stanke M, Tzvetkova A, Morgenstern B. AUGUSTUS at EGASP: using EST, protein and genomic alignments for improved gene prediction in the human genome. Genome Biol. 2006;7:11.

54. Angiuoli SV, Salzberg SL. Mugsy: fast multiple alignment of closely related whole genomes. Bioinformatics. 2011;27(3):334-42.

55. Fouts DE, Brinkac L, Beck E, Inman J, Sutton G. PanOCT: automated clustering of orthologs using conserved gene neighborhood for pangenomic analysis of bacterial strains and closely related species. Nucleic Acids Res. 2012;40(22):e172.

56. Nelson CE, Hersh BM, Carroll SB. The regulatory content of intergenic DNA shapes genome architecture. Genome Biol. 2004;5(4):R25.

57. Haddadi P, Ma L, Wang H, Borhan MH. Genome-wide transcriptomic analyses provide insights into the lifestyle transition and effector repertoire of Leptosphaeria maculans during the colonization of Brassica napus seedlings. Mol Plant Pathol. 2016;17(8):1196-210.

58. Park BH, Karpinets TV, Syed MH, Leuze MR, Uberbacher EC. CAZymes analysis toolkit (CAT): web service for searching and analyzing carbohydrateactive enzymes in a newly sequenced organism using CAZy database. Glycobiology. 2010;20(12):1574-84.

59. Crook GE, Hon G, Chandonia JM, Brenner SE. WebLogo: A sequence Logo generator. Genome Res. 2004;14(6):1188-90.

Ready to submit your research? Choose BMC and benefit from:

- fast, convenient online submission

- thorough peer review by experienced researchers in your field

- rapid publication on acceptance

- support for research data, including large and complex data types

- gold Open Access which fosters wider collaboration and increased citations

- maximum visibility for your research: over $100 \mathrm{M}$ website views per year

At BMC, research is always in progress.

Learn more biomedcentral.com/submissions 